\title{
Quadrature Formulas for Infinite Integrals
}

\author{
By W. M. Harper
}

1. Introduction. Since the advent of high-speed computers, "mechanical" quadratures of the type

$$
\int_{a}^{b} w(x) f(x) d x \sim \sum_{j=1}^{n} H_{j} f\left(a_{j}\right)
$$

have become increasingly important. The only quadrature generally available for the case $b=-a=\infty$ is the Hermite-Gauss formula although the LaguerreGauss formula can also be used if $f(x)$ is an even function of $x$. The latter would, however, require computation of twice the number of ordinates for a corresponding degree of precision and would therefore rarely be preferred. In either case the integrand is supposed to behave like the product of an exponential function and a polynomial. For purely algebraic integrands it would appear to be more appropriate to use a quadrature based on an algebraic weight function even though the degree of the polynomial approximation to $f(x)$ is limited.

In this paper, formulas of type (1) are derived with weight function $w(x)=$ $\left(1+x^{2}\right)^{-k-1}$ for the range $b=-a=\infty$. In a modified form they are shown to be superior to the Hermite-Gauss and Laguerre-Gauss quadratures for a particular class of statistical integrals.

2. Derivation of Quadratures. In the quadrature formula

$$
\int_{-\infty}^{\infty}\left(1+x^{2}\right)^{-k-1} f(x) d x=\sum_{j=1}^{n} H_{j} f\left(a_{j}\right)+E_{n, k}
$$

the abscissas $a_{j}$ will be the zeros of the $n$th degree polynomial $\phi_{n, k}(x)$ which satisfies the orthogonality condition

$$
\int_{-\infty}^{\infty}\left(1+x^{2}\right)^{-k-1} \phi_{m, k}(x) \phi_{n, k}(x) d x=0, \quad(m \neq n, m+n<2 k+1) .
$$

By standard methods given for example in [2], [4], it is easily shown from (3) that the orthogonal system of polynomials is given by the Rodrigues formula

$$
\phi_{n, k}(x)=(-1)^{n} \frac{\Gamma(2 k-2 n+2)}{\Gamma(2 k-n+2)}\left(1+x^{2}\right)^{k+1} \frac{d^{n}}{d x^{n}}\left(1+x^{2}\right)^{n-k-1}, \begin{aligned}
& \\
& \quad(n<k+1)
\end{aligned}
$$

where the standardizing constant is chosen to make the coefficient of $x^{n}$ unity. By direct manipulations with (4) and repeated use of Leibnitz' formula, the recurrence relations (5)-(10) are easily established. They are

$$
\begin{gathered}
\phi_{n+1, k}(x)=x \phi_{n, k}(x)-\frac{n(2 k-n+2)}{(2 k-2 n+1)(2 k-2 n+3)} \phi_{n-1, k}(x), \\
\left(1+x^{2}\right) \phi_{n, k}^{\prime}(x)=(2 k-n+1) x \phi_{n, k}(x)-(2 k-2 n+1) \phi_{n+1, k}(x),
\end{gathered}
$$

Received August 28, 1961. 


$$
\begin{aligned}
& \left(1+x^{2}\right) \phi_{n, k}^{\prime}(x)=n x \phi_{n, k}(x)+\frac{n(2 k-n+2)}{2 k-2 n+3} \phi_{n-1, k}(x), \\
& \phi_{n, k+1}(x)=\frac{2 k-2 n+3}{(2 k-n+2)(2 k-n+3)} \\
& \cdot\left[\left\{(4 k-2 n+3)+(2 k-2 n+1) x^{2}\right\} \phi_{n, k}(x)\right. \\
& \left.-(2 k-2 n+1)\left(1+x^{2}\right) \phi_{n, k-1}(x)\right] \text {. } \\
& x\left(1+x^{2}\right) \phi_{n, k}^{\prime}(x)=\left[n x^{2}-(2 k-n+2)\right] \phi_{n, k}(x) \\
& +\frac{(2 k-n+2)(2 k-n+3)}{2 k-2 n+3} \phi_{n, k+1}(x), \\
& x \phi_{n, k}^{\prime}(x)=(2 k-n+1) \phi_{n, k}(x)-(2 k-2 n+1) \phi_{n, k-1}(x) .
\end{aligned}
$$

The polynomial system can now be extended to include values of $n$ excluded in (4). For $n>k+\frac{3}{2}$ however, complex zeros make their appearance so that no useful quadratures are available for this range of $n$.

It is similarly easily shown that $\phi_{n, k}(x)$ is a solution of the differential equation

$$
\left(1+x^{2}\right) y^{\prime \prime}-2 k x y^{\prime}+n(2 k-n+1) y=0
$$

whence the relation

$$
\phi_{n, k}(x)=\left(\frac{i}{2}\right)^{n} n ! \frac{\Gamma\left(k-n+\frac{3}{2}\right)}{\Gamma\left(k+\frac{8}{2}\right)} C_{n}^{-k-1 / 2}(i x)
$$

can be established where in the notation of [1], $C_{n}{ }^{\lambda}(z)$ (designated by $P_{n}{ }^{(\lambda)}(z)$ in [7]) is the Gegenbauer or ultraspherical polynomial of degree $n$ and parameter $\lambda$. Relations with Legendre functions can also be established, namely:

$$
\begin{aligned}
& \phi_{n, k}(x)=(-1)^{n+1} \pi^{3 / 2} \lim _{s \rightarrow k} \\
& \cdot\left[2^{s-n} i^{s+n+1} \frac{\Gamma\left(s-n+\frac{3}{2}\right)}{\Gamma(2 s-n+2)} \operatorname{cosec} s \pi\left(1+x^{2}\right)^{s / 2+1 / 2} P_{s-n}^{s+1}(i x)\right]
\end{aligned}
$$

where $P_{\nu}{ }^{\mu}(z)$, in the notation of [1], is the associated Legendre function of the first kind with parameters $\mu$ and $\nu$, and

$$
\phi_{n, k}(x)=2^{k-n+1 / 2} \Gamma\left(k-n+\frac{3}{2}\right)\left(1+x^{2}\right)^{k / 2+1 / 4} \mathbf{P}_{k+1 / 2}^{-k+n-1 / 2}\left[x\left(1+x^{2}\right)^{-1 / 2}\right]
$$

where $\mathrm{P}_{\nu}{ }^{\mu}(z)$ is the associated Legendre function of the first kind with definition suitable for the cut in the real axis from $z=-1$ to $z=1$. The limit in (13) caters to integer values of $k$ (see [3]).

The weight coefficients and error term in (2) can be determined by standard methods with the results

$$
\begin{aligned}
& H_{j}=2^{2 k-2 n+2} n ! \frac{\left[\Gamma\left(k-n+\frac{3}{2}\right)\right]^{2}}{\Gamma(2 k-n+2)}\left(1+a_{j}^{2}\right)^{-1}\left[\phi_{n, k}^{\prime}\left(a_{j}\right)\right]^{-2} \\
&\left\{\begin{aligned}
E_{n, k} & =\frac{f^{(2 n)}(\xi)}{(2 n) !} \int_{-\infty}^{\infty}\left(1+x^{2}\right)^{-k-1}\left[\phi_{n, k}(x)\right]^{2} d x \\
& =\frac{2^{2 k-2 n+2} n !\left[\Gamma\left(k-n+\frac{3}{2}\right)\right]^{2}}{(2 k-2 n+1)(2 n) ! \Gamma(2 k-n+2)} f^{(2 n)}(\xi), \quad\left(n<k+\frac{1}{2}\right) .
\end{aligned}\right.
\end{aligned}
$$


The restriction on $n$ is necessary to ensure convergence of the error estimate but does not ensure that a close upper bound to the actual error can be obtained (see, for example, [2]).

For practical purposes a more convenient form of the quadrature is

$$
\int_{-\infty}^{\infty} f(x) d x \sim \sum_{j=1}^{n} K_{j} f\left(a_{j}\right)
$$

here the weight coefficients are given by

$$
K_{j}=H_{j}\left(1+a_{j}^{2}\right)^{k+1} .
$$

The values of $a_{j}$ and $K_{j}$ for four- and six-point formulas for some integral values of $k$ are given in Table 1 .

The right-hand side of (17) is a function of $k$ as well as of $n$; for a given value of $n$, therefore, there will be a value or values of $k$ depending on $f(x)$ which will give the "best" approximation to the integral on the left. The determination of such values and the corresponding parameters appears to be too formidable a task for practical applications. For the special cases $k=n-1, k=n$, however, solution of (11) with $x=\cot \theta$ enables $\phi_{n, k}(x)$ to be obtained in the forms

$$
\begin{gathered}
\phi_{n, n-1}(x)=\operatorname{cosec}^{n}(\operatorname{arc} \cot x) \cos (n \operatorname{arc} \cot x), \\
\phi_{n, n}(x)=(n+1)^{-1} \operatorname{cosec}^{n+1}(\operatorname{arc} \cot x) \sin [(n+1) \operatorname{arccot} x] .
\end{gathered}
$$

The zeros are now simple cotangents and the weight coefficients $H_{j}$ assume simple trigonometric form; the resulting quadratures can be written as

$$
\begin{array}{ll}
\int_{-\infty}^{\infty}\left(1+x^{2}\right)^{-1} f(x) d x \sim \frac{\pi}{n} \sum_{j=1}^{n} f\left[\cot \frac{(2 j-1) \pi}{2 n}\right], & (k=n-1), \\
\int_{-\infty}^{\infty}\left(1+x^{2}\right)^{-1} f(x) d x \sim \frac{\pi}{n+1} \sum_{j=1}^{n} f\left(\cot \frac{j \pi}{n+1}\right), & (k=n) .
\end{array}
$$

These formulas can also be deduced from the Chebyshev-Gauss quadratures

$$
\begin{aligned}
& \int_{-1}^{1}\left(1-y^{2}\right)^{-1 / 2} g(y) d y \sim \frac{\pi}{n} \sum_{j=1}^{n} g\left[\cos \frac{(2 j-1) \pi}{2 n}\right], \\
& \int_{-1}^{1}\left(1-y^{2}\right)^{-1 / 2} g(y) d y \sim \frac{\pi}{n+1} \sum_{j=1}^{n} g\left(\cos \frac{j \pi}{n+1}\right)
\end{aligned}
$$

by the substitutions $y=x\left(1+x^{2}\right)^{-1 / 2}, g(y)=f(x)$.

3. Practical Application. An example of a useful application for the quadratures is the evaluation of integrals arising in the determination of the statistical distribution of the ratio of two quadratic forms in normal variates. If the quadratic forms are independent mean half-square successive differences based on sample sizes of $p$ and $q$ respectively, one of the integrals which require evaluation can be written in the form

$$
I(z)=\int_{-\infty}^{\infty}\left(1+x^{2}\right)^{-1} \prod_{r=2}^{p-1}\left(a_{r}^{2}+x^{2}\right)^{-1 / 2} \prod_{s=1}^{q-1}\left(1+b_{s}{ }^{2} z^{-1}+x^{2}\right)^{-1 / 2} d x
$$


TABLE 1

Abscissas and Weights for Quadrature (17)

A. $n=4$

\begin{tabular}{c|cc|cc}
\hline \multirow{2}{*}{$k$} & \multicolumn{2}{|c|}{ $\pm a_{j}$} & \multicolumn{2}{|c}{$K_{j}$} \\
\hline \multirow{2}{*}{4} & 0.41421 & 35624 & 0.92015 & 11845 \\
& 2.41421 & 35624 & 5.36303 & 41227 \\
5 & 0.32491 & 96962 & 0.69465 & 18830 \\
& 1.37638 & 19205 & 1.81862 & 22399 \\
6 & 0.27618 & 30252 & 0.58086 & 65620 \\
& 1.06005 & 79874 & 1.17945 & 11502 \\
7 & 0.24436 & 83118 & 0.50932 & 47880 \\
& 0.89298 & 76737 & 0.90816 & 46087 \\
8 & 0.22150 & 78137 & 0.45903 & 94023 \\
& 0.78587 & 59159 & 0.75578 & 97944 \\
9 & 0.20405 & 97869 & 0.42121 & 27662 \\
& 0.70979 & 86678 & 0.65698 & 70999 \\
10 & 0.19017 & 76238 & 0.39142 & 46836 \\
& 0.65220 & 46710 & 0.58705 & 73261 \\
& 0.17879 & 14705 & 0.36717 & 90805 \\
& 0.60665 & 77372 & 0.53455 & 96626 \\
\hline
\end{tabular}

B. $n=6$

\begin{tabular}{|c|c|c|c|c|}
\hline$k$ & \multicolumn{2}{|c|}{ $\pm a_{j}$} & \multicolumn{2}{|c|}{$K_{j}$} \\
\hline 5 & $\begin{array}{l}0.26794 \\
1.00000 \\
3.73205\end{array}$ & $\begin{array}{l}91924 \\
00000 \\
08076\end{array}$ & $\begin{array}{l}0.56119 \\
1.04719 \\
7.81638\end{array}$ & $\begin{array}{l}14763 \\
75512 \\
89333\end{array}$ \\
\hline 6 & $\begin{array}{l}0.22824 \\
0.79747 \\
2.07652\end{array}$ & $\begin{array}{l}34744 \\
33889 \\
13966\end{array}$ & $\begin{array}{l}0.47217 \\
0.73421 \\
2.38399\end{array}$ & $\begin{array}{l}91694 \\
88392 \\
35955\end{array}$ \\
\hline 7 & $\begin{array}{l}0.20219 \\
0.68370 \\
1.57850\end{array}$ & $\begin{array}{l}80919 \\
47228 \\
04858\end{array}$ & $\begin{array}{l}0.41550 \\
0.58969 \\
1.44716\end{array}$ & $\begin{array}{l}76425 \\
00381 \\
80133\end{array}$ \\
\hline 8 & $\begin{array}{l}0.18342 \\
0.60816 \\
1.31884\end{array}$ & $\begin{array}{l}80037 \\
30047 \\
38384\end{array}$ & $\begin{array}{l}0.37535 \\
0.50404 \\
1.06492\end{array}$ & $\begin{array}{l}93234 \\
67421 \\
43997\end{array}$ \\
\hline 9 & $\begin{array}{l}0.16907 \\
0.55326 \\
1.15411\end{array}$ & $\begin{array}{l}35256 \\
32106 \\
46518\end{array}$ & $\begin{array}{l}0.34499 \\
0.44635 \\
0.85743\end{array}$ & $\begin{array}{l}40643 \\
57833 \\
60559\end{array}$ \\
\hline 10 & $\begin{array}{l}0.15763 \\
0.51101 \\
1.03809\end{array}$ & $\begin{array}{l}63749 \\
94490 \\
74230\end{array}$ & $\begin{array}{l}0.32098 \\
0.40432 \\
0.72680\end{array}$ & $\begin{array}{l}68394 \\
69556 \\
65190\end{array}$ \\
\hline
\end{tabular}


TABLE 2

Comparison of Quadrature Formulas in Evaluating I(1)

\begin{tabular}{|c|c|c|c|c|c|}
\hline \multirow{2}{*}{$\begin{array}{l}\text { Quadrature } \\
\text { Series }\end{array}$} & \multirow{2}{*}{$\frac{\text { No. Abscissas }}{-}$} & \multicolumn{2}{|c|}{ Result } & \multicolumn{2}{|c|}{$E \times 10^{8}$} \\
\hline & & 1.2106 & 5423 & & - \\
\hline Algebraic, $k=j$ & 6 & 1.2106 & 4384 & & 1039 \\
\hline Algebraic, $k=6$ & 6 & 1.2106 & 5381 & & 42 \\
\hline Algebraic, $k=7$ & 6 & 1.2106 & 5415 & & 8 \\
\hline Algebraic, $k=8$ & 6 & 1.2081 & 0423 & 25 & 5000 \\
\hline Algebraic, $k=9$ & 6 & 1.2025 & 0816 & 81 & 4607 \\
\hline Algebraic, $k=10$ & 6 & 1.1942 & 4044 & 164 & 1379 \\
\hline Hermite & 6 & 1.1610 & 8623 & 495 & 6800 \\
\hline Hermite & 8 & 1.1879 & 0738 & 227 & 4685 \\
\hline Hermite & 10 & 1.1994 & 3337 & 112 & 2086 \\
\hline Laguerre & 6 & 1.1674 & 2007 & 432 & 3416 \\
\hline
\end{tabular}

where the $a_{r}$ and $b_{s}$ are constants. In order to compare methods (25) was evaluated by various quadratures for the case $p=4, q=3, z=1$ when the test integral becomes

$$
\begin{aligned}
& I(1)=\int_{-\infty}^{\infty}\left(1+x^{2}\right)^{-1} {\left[\left(\frac{1}{2} \sqrt{2}+x^{2}\right)\left(2 \sqrt{2}-2+x^{2}\right)\right.} \\
&\left.\cdot\left\{\frac{1}{3}(7-2 \sqrt{2})+x^{2}\right\}\left\{\frac{1}{9}(13-2 \sqrt{2})+x^{2}\right\}\right]^{-1 / 2} d x .
\end{aligned}
$$

The quadrature (17) was applied for the values $k=5(1) 10$ using six abscissas in each case. The Hermite-Gauss quadrature was used with six, eight and ten abscissas, and the Laguerre-Gauss formula for six abscissas (which requires the same number of evaluations of the integrand as the other formulas for twelve abscissas but which is of degree of precision eleven as against twenty-three for the others). The abscissas and weights for the Hermite formula were taken from the values tabulated in [6] and those for the Laguerre method from [5]. The results together with the correct value of $I(1)$ determined by a series method are tabulated to eight decimal places in Table 2 which also shows the errors of the methods.

The table shows the superiority of the "algebraic" quadratures over the Hermite and Laguerre formulas for this integral; even the use of ten abscissas for the Hermite quadrature leaves an error much greater than the algebraic quadratures with only six abscissas except for the case $k=10$. The best algebraic quadrature is for $k=7$ but the advantage over those for $k=5$ and $k=6$ is too small to compensate for the simplicity of the latter two cases when used in the equivalent forms shown in (21) and (22) respectively. In addition, the quadrature (22) evaluates $I$ (1) correctly to eight decimal places for $n=8$ as does (21) for $n=9$.

4. Acknowledgements. The author is indebted to Mr. J. A. Macdonald for some helpful suggestions and to Miss Primula Thomson for much of the computing. 
The paper is published with the permission of the Chief Scientist, Department of Supply, Australian Defence Scientific Service, Melbourne, Victoria, Australia.

Department of Supply

Australian Defence Scientific Service

Defence Standards Laboratories

Maribyrnong, Victoria, Australia.

1. A. Erdelyi, et al., Higher Transcendental Functions, McGraw-Hill, New York, 1953, v. 1, p. $120-181$, v. 2 , p. $174-178$.

2. F. B. Hildebraxd, Introduction to Numerical Analysis, McGraw-Hill, New York, 1956, p. $312-367$

3. E. W. Hobson, Spherical and Ellipsoidal Harmonics, Cambridge University Press, 1931, p. 178-292.

4. Z. Kopal, Numerical Analysis, Wiley \& Sons, New York, 1955, p. 347-439.

5. H. E. SAlzer \& R. ZUCKER, "Table of the zeros and weight factors of the first fifteen Laguerre polynomials," Bull. Amer. Math. Soc. v. 55, 1949, p. 1004-1012.

6. H. E. Salzer, R. Zrcker \& R. Capuano, "Table of the zeros and weight factors of the first twenty Hermite polynomials," J. Res. Nat. Bur. Standards, v. 48, 1952, p. 111-116.

7. G. Szegö, Orthogonal Polynomials, American Ilathematical Society Colloquium Publications, v. 23,1959, p. 81-86. 